\title{
Efecto de la Suplementación con una Mezcla Probiótica sobre el Comportamiento Productivo de Cuyes (Cavia porcellus) durante la Fase de Crecimiento y Acabado
}

\author{
Effect of a Probiotic Mix Supplementation on the Productive Performance of \\ Growing and Finishing Guinea Pigs (Cavia porcellus)
}

Javier Cano W. ${ }^{1}$, Fernando Carcelén C. ${ }^{1,5}$, Miguel Ara G. ${ }^{1}$, Willian Quevedo G. ${ }^{3}$, Arnaldo Alvarado S. ${ }^{2}$, Ronald Jiménez A. ${ }^{4}$

\section{Resumen}

\begin{abstract}
Se evaluó el efecto de la adición de una mezcla probiótica en la dieta sobre la ganancia de peso, consumo de alimento y conversión alimenticia de cuyes en crecimiento y engorde. Se trabajó con 64 cuyes machos de un día de edad de una granja experimental en Huancayo, Perú. Los animales fueron distribuidos al azar en cuatro tratamientos consistentes en una dieta base de forraje (ryegrass-trébol rojo) suplementada con afrechillo de trigo, más 0, 100, 150 o $200 \mathrm{ml}$ de una mezcla probiótica en suspensión de LactobacillusBifidobacterium-Saccharomyces. La suspensión probiótica fue mezclada con $1 \mathrm{~kg}$ del suplemento y administrada por 10 semanas. Se observaron patrones significativos de respuesta lineal a los niveles del probiótico en ganancia de peso $(y=631.04571+0.60537 x$; $\left.\mathrm{r}^{2}=0.2971, \mathrm{p}=0.029\right)$ y conversión alimenticia $\left(y=6.21121-0.0339 \mathrm{x} ; \mathrm{r}^{2}=0.625, \mathrm{p}=0.0003\right)$, sin que hubiera una respuesta significativa en consumo total de alimento. Se concluye que la mezcla probiótica tiene el potencial de incrementar la productividad y la eficiencia alimenticia en cuyes en crecimiento y acabado; sin embargo, mayores niveles del probiótico deberían ser explorados para obtener resultados biológicos y prácticos óptimos.
\end{abstract}

Palabras clave: cuy, probióticos, parámetros productivos

\section{Abstract}

The study evaluated the effect of addition of a probiotic mix in the diet on body weight gain, feed intake and feed conversion of growing and finishing guinea pigs. A total of 64 male guinea pigs, 1 day-old from an experimental farm in Huncayo, Peru were

\footnotetext{
${ }^{1}$ Laboratorio de Bioquímica, Nutrición y Alimentación Animal, ${ }^{2}$ Laboratorio de Patología Clínica y Biología Molecular, ${ }^{3}$ Laboratorio de Zootecnia y Producción Agropecuaria, Facultad de Medicina Veterinaria, Universidad Nacional Mayor de San Marcos, Lima, Perú

${ }^{4}$ Estación Experimental del Centro de Investigación IVITA-El Mantaro, Universidad Nacional Mayor de San Marcos, Huancayo, Perú

${ }^{5}$ E-mail: nandodeme@gmail.com
}

Recibido: 16 de marzo de 2015

Aceptado para publicación: 22 de agosto de 2015 
selected. The animals were randomly allocated to four feed treatments consisting of a rye grass-clover forage basal diet supplemented with wheat bran plus $0,100,150$ or $200 \mathrm{ml}$ of a Lactobacillus-Bifidobacterium-Saccharomyces probiotic mix suspension. The probiotic was mixed with $1 \mathrm{~kg}$ of the supplement and supplied during 10 weeks. Significant linear response patterns to the probiotic levels were observed for body weight gain $(y=631.04571$ $\left.+0.60537 x ; \mathrm{r}^{2}=0.2971, \mathrm{p}=0.029\right)$ and feed conversion $\left(y=6.21121-0.0339 \mathrm{x} ; \mathrm{r}^{2}=0.625\right.$, $\mathrm{p}=0.0003$ ). There was no significant effect on feed intake. It is concluded that the probiotic mix has the potential for improving the productivity and feed efficiency of guinea pigs, but higher levels should be explored in order to obtain biological and practical optimal results.

Key words: guinea pig, probiotics, production parameters

\section{INTRODUCCIÓN}

Uno de los mayores riesgos sanitarios en las crianzas familiar-comercial y comercial de cuyes es la ocurrencia de enfermedades infecciosas, especialmente la salmonelosis (Ameghino, 1968; Aguilar et al., 2011); enfermedad hacia la cual los cuyes parecen tener particular susceptibilidad, con tasas de morbilidad de hasta $53 \%$ en lactantes, $20 \%$ en recría y $31 \%$ en adultos (Chauca, 1997).

Los antibióticos son ampliamente utilizados como medida preventiva para el control de enfermedades en explotaciones de bajo riesgo. Desafortunadamente, esta práctica es aplicada en forma indiscriminada, particularmente en lo que concierne a productos, dosis y estrategias de aplicación, generando un potencial problema de salud pública, debido a los posibles residuos de antibióticos en los productos y subproductos animales, así como por el riesgo de generación de resistencia a los antibióticos (Chauca, 1995).

Los inconvenientes asociados al uso de antibióticos en forma preventiva o como promotores de crecimiento han motivado la búsqueda de nuevas opciones de prevención y control de problemas sanitarios gastrointestinales, siendo una de las más promisorias el uso de probióticos. Estos han sido definidos como «microorganismos vivos, que al ser consumidos o administrados en cantidades adecuadas como parte del alimento confieren un beneficio sanitario al hospedero» (FAO/WHO, 2001).

Entre los organismos probióticos más comunes figuran Lactobacillus, Streptococcus, Enterococcus, Bacillus y Saccharomyces (Preidis et al., 2011) y el efecto benéfico ha sido asociado a tres mecanismos aparentes: (a) exclusión competitiva por el substrato y sitios de adhesión del epitelio intestinal, (b) secreción de sustancias antimicrobiales, y (c) estimulación de la inmunidad del hospedero (Zihler et al., 2011). Estos mecanismos parecen explicar no solo un efecto positivo general de los probióticos sobre un gran número de condiciones gastrointestinales en humanos (Ritchie y Romanuk, 2012), sino también sobre el comportamiento productivo de especies animales como pollos (Rodríguez, 1994), cerdos (Jurgens et al., 1997) y conejos (Zoccarato et al., 1995; Ayyat et al., 1996).

Dado el riesgo latente de salmonelosis en crianzas de cuyes, es razonable explorar el potencial que tienen los probióticos para mejorar el comportamiento productivo del cuy. Con este fin, se diseñó este estudio dirigido a probar el efecto de una mezcla probiótica comercial sobre la ganancia de peso, consumo y conversión alimenticia de cuyes en un sistema comercial en el valle del Mantaro, Perú. 


\section{Materiales y Métodos}

El estudio se realizó entre agosto de 2009 y enero de 2010 en la Estación Experimental El Mantaro del Instituto Veterinario de Investigaciones Tropicales y de Altura (IVITA). La estación está a $34 \mathrm{~km}$ de la ciudad de Huancayo, en el distrito de El Mantaro, provincia de Jauja, departamento de Junín, a una altitud de 3320 msnm y con temperatura y precipitación media anual de $10.9{ }^{\circ} \mathrm{C}$ y $750 \mathrm{~mm}$, respectivamente.

Se trabajó con 64 cuyes hembras de la línea Cárnica, línea obtenida en el Centro de Investigación IVITA mediante selección genética para conversión alimenticia (Jiménez y Huamán, 2010). Las hembras se encontraban en el último tercio de gestación y fueron distribuidas al azar en cuatro tratamientos, los cuales recibieron $0,100,150$ y $200 \mathrm{ml}$ de una suspensión probiótica comercial.

Una semana antes de la fecha probable de parto, las hembras fueron alojadas individualmente en pozas de maternidad de $1 \times 1.14 \times 0.74 \mathrm{~m}$, previamente desinfectadas, acondicionadas con una cama de paja de cebada de $10 \mathrm{~cm}$ de espesor y equipadas con comederos y bebederos de arcilla. Las hembras fueron alimentadas dos veces al día con afrechillo de trigo a una razón diaria de $4 \%$ del peso corporal y con una mezcla de partes iguales de los forrajes rye grass italiano (Lolium perenne) y trébol rojo (Trifolium pratense), suministrado en el piso y a razón diaria de 6\% (base seca) del peso corporal. El forraje proporcionado era cosechado el día previo al suministro. El agua fue ofrecida $a d$ libitum en bebederos de arcilla, reemplazándose la totalidad del contenido cada día. La composición químico-nutricional de los alimentos se muestra en el Cuadro 1.

Como fuente del probiótico se usó la suspensión comercial «Biomodulador Oral/ Soluble» (Reinmark SRL, Perú), cuya composición volumétrica es de $30 \%$ de Lactobacillus acidophilus, L. casei,
Cuadro 1. Valores del análisis proximal de la mezcla forrajera rye grass italiano (Lolium perenne) - trébol rojo (Trifolium pratense) y de afrechillo de trigo en base seca $^{1}$

\begin{tabular}{lcc}
\hline Componentes & $\begin{array}{c}\text { Mezcla } \\
\text { forrajera } \\
(\%)\end{array}$ & $\begin{array}{c}\text { Afrechillo } \\
(\%)\end{array}$ \\
\hline Proteína cruda & 21.23 & 20.92 \\
Extracto etéreo & 2.37 & 2.96 \\
Fibra cruda & 14.26 & 9.19 \\
$\begin{array}{l}\text { Cenizas } \\
\begin{array}{l}\text { Extracto libre de } \\
\text { nitrógeno }\end{array}\end{array}$ & 8.95 & 5.62 \\
\hline
\end{tabular}

${ }^{1}$ Mezcla forrajera: 24.06 de MS ; afrechillo: $94.35 \%$ de MS

Bifidobacterium longum, Saccharomyces cerevisiae y $S$. boulardi, todas a $10^{10}$ bacterias $\mathrm{ml}^{-1}$, y $70 \%$ de ácido cítrico $25 \%$. Los volúmenes de suspensión correspondientes a cada tratamiento fueron diluidos a $300 \mathrm{ml}$ de agua y mezclados con $1 \mathrm{~kg}$ del afrechillo.

A partir del tercer día del parto y por cinco días consecutivos, las madres recibieron la suplementación probiótica correspondiente a los tratamientos. En este mismo lapso, las crías macho también recibieron la suspensión probiótica mediante administración diaria oral de $1 \mathrm{ml}$ de la suspensión diluida por individuo, usando una microjeringa.

Al destete (día 16 después del parto), se seleccionó al azar una cría macho por madre para formar grupos de 16 crías machos por tratamiento. En la selección se tomó en consideración la proximididad de las fechas de nacimiento. Los cuyes se distribuyeron en pozas de crecimiento-engorde de $2 \times$ $1.2 \times 0.74 \mathrm{~m}$, a razón de cuatro animales por poza. Las pozas fueron acondicionadas y equipadas de manera semejante a las pozas de maternidad. Ese mismo día se reinició la aplicación de los tratamientos probióticos 
respectivos a los machos destetados, también por cinco días consecutivos y en las mismas dosis aplicadas a las crías antes del destete. Esta suplementación se repitió partir del día 46 posparto.

El programa de suplementación al tercer día de edad persigue procurar la siembra de las bacterias benéficas del probiótico en el intestino del recién nacido, el cual en los primeros días es estéril o muy poco colonizado y evitar que las bacterias patógenas colonicen el intestino. La aplicación a los 16 días de edad es coincidente con el destete, que es cuando hay cambios en la dieta, estrés en el animal y cambios en la microbiota intestinal, y procura renovar la siembra de las bacterias probióticas, desplazando a las patógenas o potencialmente patógenas que pueden aprovechar los cambios intestinales. La aplicación a los 46 días persigue propiciar mejores condiciones intestinales para iniciar un desarrollo y engorde del animal en la última parte de la cría.

El manejo de los machos fue concurrente con el sistema de cría actualmente en uso en la estación. La mezcla de forrajes, previamente pesada, se suministró dos veces al día (8:30 y 14:00) a razón de 6\% del peso corporal de los animales. El afrechillo se administró una vez al día en las mañanas $(8: 30)$ y representó el $4 \%$ del peso corporal. El alimento rechazado fue pesado semanalmente. Se tomaron muestras semanales de forraje ofrecido y rechazado con la finalidad de determinar el contenido de materia seca y el consumo de la misma.

Se evaluó la ganancia de peso vivo, consumo de alimento y conversión alimenticia. El peso vivo fue controlado individualmente, antes del suministro del alimento, cada 9 días durante 55 días posdestete. La ganancia de peso fue calculada como la diferencia entre el peso final y el inicial. El consumo de materia seca se determinó mediante el peso diario del afrechillo y forraje y el peso semanal del residuo, ambos expresados como materia seca. El consumo por poza se calculó como la diferencia entre la materia seca suministrada y la materia seca residual. La conversión alimenticia para cada poza se calculó como la relación entre el consumo total de materia seca y la ganancia total de peso vivo. En todos los casos se usó una balanza electrónica de $1 \mathrm{~g}$ de precisión.

El efecto de los diferentes niveles del probiótico sobre las variables de respuesta fue estimado mediante análisis de varianza para un modelo completamente al azar con cuatro tratamientos y cuatro repeticiones. El patrón de respuesta al probiótico fue estimado mediante contrastes lineal y cuadrático y la función de respuesta mediante análisis de regresión (Steel et al., 1997). El nivel de significancia usado para las pruebas de hipótesis fue 0.05 . Los análisis estadísticos se llevaron a cabo con ayuda del paquete SAS/ STAT® 9.2 (SAS Institute, 2009).

\section{Resultados y Discusión}

\section{Ganancia de Peso}

Se evidenció un patrón de respuesta lineal a los niveles del probiótico $(\mathrm{p}=0.0242)$; sin embargo, no se pudo evidenciar un patrón cuadrático $(\mathrm{p}=0.4115) \mathrm{y}$, por lo tanto, tampoco se pudo obtener una respuesta máxima. La función de respuesta lineal al probiótico fue de $y=631.04571+0.60537 x\left(r^{2}=0.2971\right.$, $\mathrm{p}=0.029$ ), donde $y$ es la ganancia de peso y $x$ son los niveles del probiótico (Fig. 1). Los resultados son concurrentes con los obtenidos por otros autores en conejos (Zoccarato et al., 1995; Ayyat et al., 1996; Bielanski et al., 1997), quienes reportaron que las dietas con Saccharomyces tuvieron mayor ganancia de peso diaria y total que el control. En el cerdo también se ha demostrado que la inclusión de levaduras en la dieta puede incrementar la ganancia de peso durante el crecimiento y mejorar la eficiencia alimenticia, sin incrementar el consumo de alimento (Jurgens et al., 1997). 


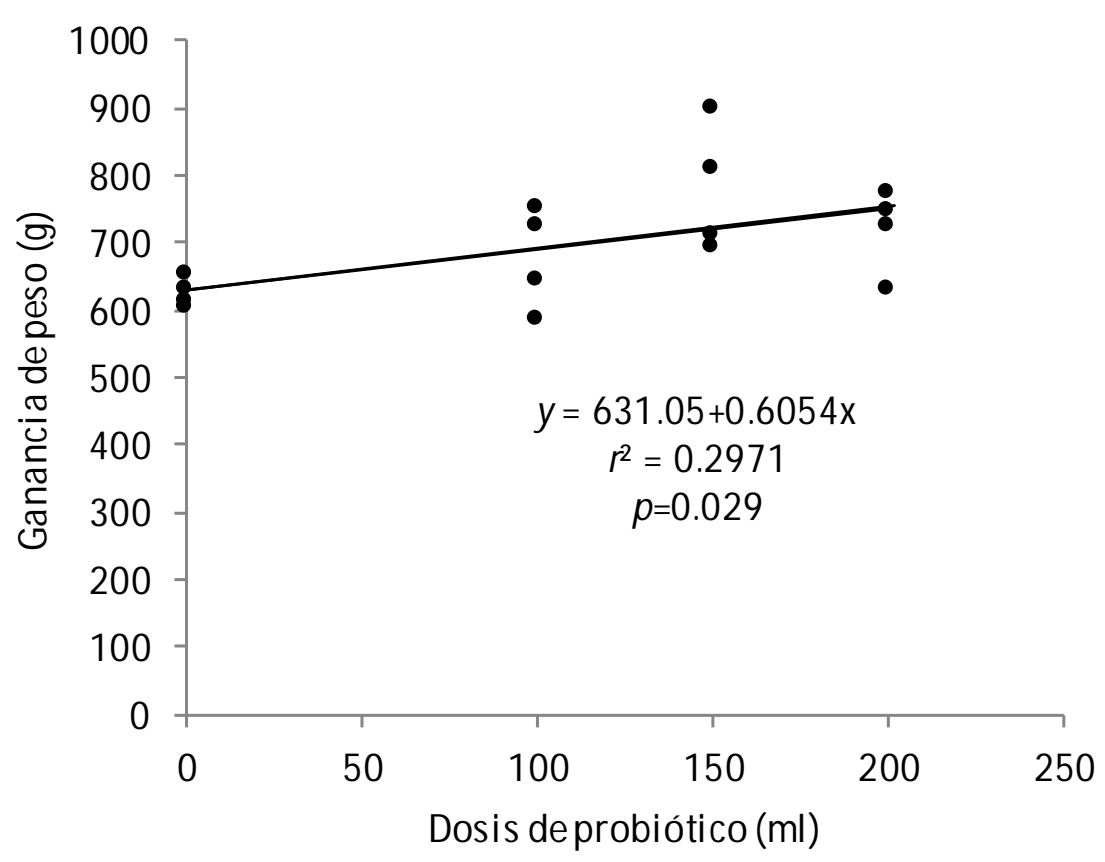

Figura 1. Ganancia de peso de cuyes durante el crecimiento y engorde como respuesta a tres dosis de probiótico. Mantaro, Huancayo, Perú

Por otro lado, algunas investigaciones han mostrado una pobre o nula respuesta a la adición de probióticos. Así, en una de las pocas referencias en cuyes, Molina (2008) no encontró respuestas significativas a la adición de probióticos de Lactobacillus acidophilus o Bacillus subtilis, ni Kustos et al. (2004) al agregar B. subtilis y B. licheniformis a la dieta de conejos. En forma similar, Gippert et al. (1992) solo encontraron un aumento de $3 \%$ en la ganancia de peso diario al incluir el probiótico (BIoplus $2 \mathrm{~B}^{\circledR}$ ), en tanto que Maertens et al. (1994) reportaron una mejora relativa en la ganancia de peso en cabras.

\section{Consumo de Materia Seca}

El incremento de los niveles del probiótico hasta $200 \mathrm{ml}$ de la suspensión no produjo efectos significativos sobre el consumo total de materia seca $(\mathrm{p}=0.3475$ para la respuesta lineal; $\mathrm{p}=0.5931$ para la respuesta cuadrática). Los patrones de respuesta se muestran en la Fig. 2.

Los estudios acerca del efecto de los probióticos sobre el consumo de alimento han tenido resultados variables. Ayyat et al. (1996) obtuvieron un mayor consumo de materia seca total con probióticos a base de Lactobacillus y Saccharomyces en dietas para conejos. Molina (2008), por otro lado, obtuvo un menor consumo con la adición de $50 \mathrm{mg} / \mathrm{kg}$ de B. subtilis en cuyes, pero mayores con $50 \mathrm{mg} / \mathrm{kg}$ de $L$. acidophilus, aunque sin diferencia estadística con el grupo control. Asimismo, Zoccarato et al. (1995) y Jurgens et al. (1997) obtuvieron aumentos de peso y mejoras de la conversión de alimento en conejos con B. subtilis y en cerdos con un suplemento de levadura, respectivamente, pero sin cambios en la ingesta de alimento. 


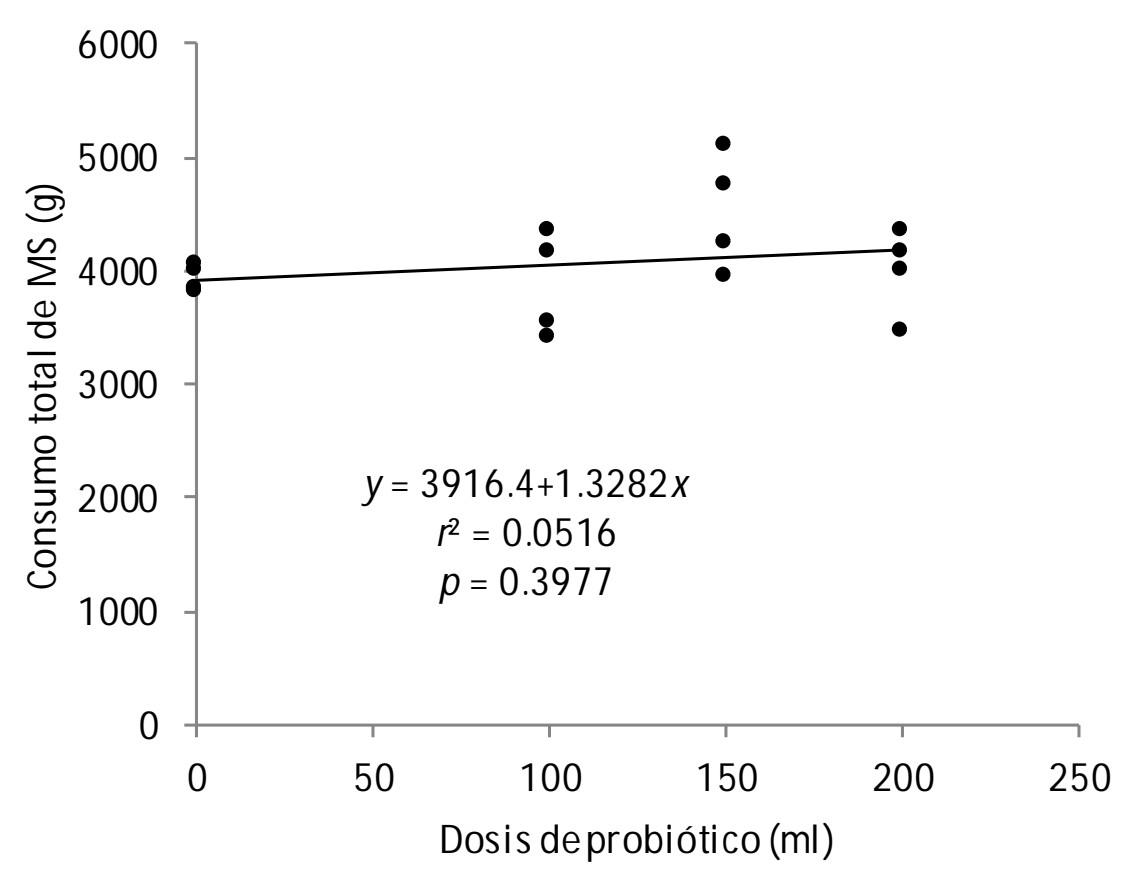

Figura 2. Consumo total de materia seca de cuyes durante el crecimiento y acabado como respuesta a tres dosis de probiótico. Mantaro, Huancayo, Perú

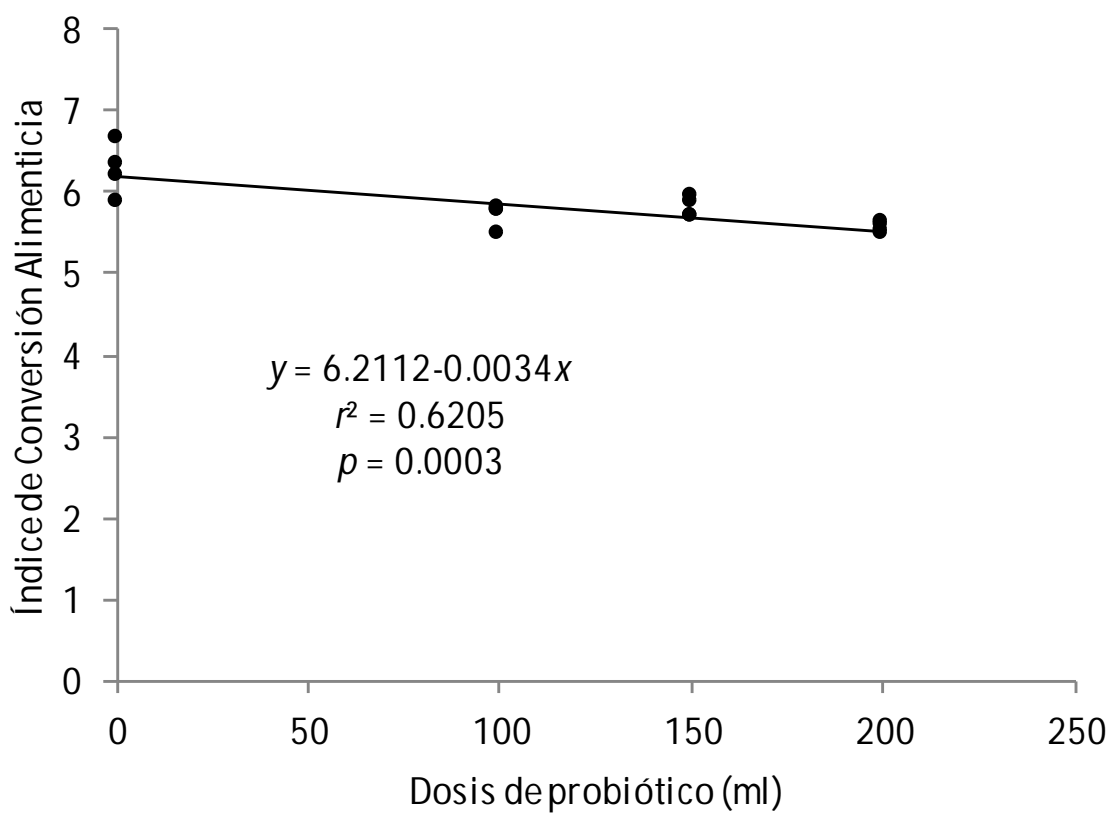

Figura 3. Índice de conversión alimenticia de cuyes durante el crecimiento y acabado como respuesta a tres dosis de probiótico. Mantaro, Huancayo, Perú 
En trabajos en pollos, Jadamus et al. (2001) reportaron que la inclusión de $B$. toyoi en las dietas de broilers permitió reducir el consumo de materia seca, pero Baidya et al. (1994) obtuvo similares consumos con la adición de B. toyoi en la dieta que el grupo control no suplementado.

\section{Conversión Alimenticia}

Los niveles del probiótico redujeron significativamente $(\mathrm{p}=0.0002)$ el índice de conversión alimenticia, posiblemente como producto de una mejor ganancia de peso; sin embargo, y como en el caso de la ganancia de peso, no se obtuvo una respuesta cuadrática $(\mathrm{p}=0.2702)$ y, por lo tanto, no se observaron respuestas mínimas. La función de respuesta lineal al probiótico fue $y=$ $6.21121-0.0339 \mathrm{x}\left(\mathrm{r}^{2}=0.625, \mathrm{p}=0.0003\right)$, donde $y$ es el índice de conversión alimenticia y $x$ son los niveles del probiótico (Fig. 3).

Estos resultados concuerdan con estudios en conejos (Zoccarato et al., 1995) y cerdos (Jurgens et al., 1997) quienes encontraron mejoras en la eficiencia alimenticia como producto de la adición de probióticos. Asimismo, Rodríguez (1994) utilizando $L$. acidophilus y Streptococcus faecium en la dieta de pollos de tres semanas obtuvieron $7 \%$ de reducción del índice de conversión alimenticia, y Ramírez et al. (2005) reportaron $12 \%$ de mejora al incluir Lactobacillus.

Los resultados de este estudio sugieren un efecto positivo del probiótico a niveles entre 0 y $200 \mathrm{ml}$ de suspensión sobre la ganancia de peso y la eficiencia alimenticia de cuyes en crecimiento y engorde; sin embargo, no se observó una inflexión en la curva de respuesta, lo cual indicaría la necesidad de ensayar niveles más altos del probiótico.

\section{Conclusiones}

- La inclusión de una mezcla probiótica en suspensión de Lactobacillus-Bifido-
bacterium-Saccharomyces en la dieta mejora la ganancia de peso y el índice de conversión alimenticia en la etapa de crecimiento y acabado de cuyes.

- El consumo de materia seca no se vio afectado por la suplementación del probiótico.

\section{Literatura Citada}

1. Aguilar G, Bustamante J, Bazán B, Falcón N. 2011. Diagnóstico situacional de la crianza de cuyes en una zona de Cajamarca. Rev Inv Vet Perú 22: 9-14. doi: 10.15381/rivep.v22i1.113

2. Ameghino CE. 1968. Sobre un brote de salmonelosis en cuyes (Cavia cobaya). Bol Ext IVITA (Perú) 3: 260-261.

3. Ayyat M, Marai I, El-Aasar T. 1996. New Zealand white rabbit does and their growing offsprings as affected by diets containing different protein level with or without Lacto-Sacc supplementation. World Rabbit Sci. 4:225-230. doi: 10.4995/wrs.1996.299

4. Baidya N, Mandal L, Sarkar SK, Banerjee GC. 1994. Combined feeding of antibiotic and probiotic on the performance of broiler. Indian Poultry Sci 29: 228-231.

5. Bielanski P, Niedzwizadek S, Zajac J, Kowalska D. 1997. Effect of probiotics on fattening and slaughter performance of rabbits. World Rabbit Sci 5: 133.

6. Chauca L. 1995. Sistemas de producción de cuyes. En: Serie Guía Didáctica: Crianza de cuyes. Lima: INIA. p 77-85.

7. Chauca L. 1997. Producción de cuyes (Cavia porcellus). Roma: Organización de las Naciones Unidas para la Agricultura y la Alimentación - FAO. $134 \mathrm{p}$.

8. FAO/WHO. 2001. Health and nutrition properties of probiotics in food including powder milk with live lactic acid bacteria. Rome: FAO. 34 p.

9. Gippert T, Virág G, Nagy I. 1992. Lacto-Sacc in rabbit nutrition. J Appl Rabbit Res 15: 1101-1104. 
10. Jadamus A, Vahjen W, Simon WV. 2001. Growth behaviour of a spore forming probiotic strain in the gastrointestinal tract of broiler and piglets. Arch Tierernahr 54: 1-17.

11. Jiménez R, Huamán A. 2010. Cuyes genéticamente geniales: manual para el manejo de reproductores híbridos especializados en producción de carne. Subproyecto desarrollo y evaluación de reproductores para maximizar la producción de cuyes de carne. Huancayo, Perú: UNMSM-INCAGRO. 175 p.

12. Jurgens M, Rikabi R, Zimmerman D. 1997. The effect of dietary active dry yeast supplement on performance of sows during gestation-lactation and their pigs. J Anim Sci 75: 593-597.

13. Kustos K, Kovács D, Gódor-Surmann K, Eiben Cs. 2004. Effect of probiotic Bioplus $2 \mathrm{~B}^{\circledR}$ on the performance of growing rabbit. En: $8^{\text {th }}$ World Rabbit Congress. Puebla, México.

14. Maertens $L$, van Renterghem $R, D e$ Groote G. 1994. Effects of dietary inclusion of Paciflor ${ }^{\circledR}$ (Bacillus CIP 5832) on the milk composition and performances of does and on caecal and growth parameters of their weanlings. World Rabbit Sci 2: 67-73. doi: 10.4995/ wrs. 1994.220

15. Molina M. 2008. Efecto probiótico de Lactobacillus acidophilus y Bacillus subtilis en cuyes (Cavia porcellus) de engorde. Tesis de Ingeniero Agropecuario. Sangolqui, Ecuador: Escuela Politécnica del Ejército. 118 p.

16. Preidis GA, Hill C, Guerrant RL, Ramakrishna BS, Tannock $G W$, Versalovic J. 2011. Probiotics, enteric and diarrheal diseases, and global health.
Gastroenterology 140: 8-14. doi: 10.1053/ j.gastro.2010.11.010

17. Ramírez B, Zambrano O, Ramírez Y, Rodríguez V. 2005. Evaluación del efecto probiótico de Lactobacillus spp origen aviar en pollitas de inicio reemplazo de la ponedera comercial en los primeros 42 días de edad. REDVET 6(9). [Internet]. Disponible en: http:// www.veterinaria.org/revistas/redvet/ n090905/090512.pdf

18. Ritchie ML, Romanuk TN. 2012. A meta-analyisis of probiotic efficacy for gastrointestinal diseases. PLoS ONE 7(4): e-34938. doi: 10.1371/journal. pone. 0034938

19. Rodríguez M. 1994. Bacterias productoras de ácido láctico: efecto sobre el crecimiento y la flora intestinal de pollos, gazapos y lechones. Tesis Doctoral. Madrid, España: Univ Complutense de Madrid. $193 \mathrm{p}$.

20. SAS Institute Inc. 2009. SAS/STAT ${ }^{\circledR}$ 9.2 User's Guide $2^{\text {nd }}$ ed. Cary, NC. 870 p.

21. Steel RGD, Torrie JH, Dickey DA. 1997. Principles and procedures of statistics: a biometrical approach. New York, USA: WCB McGraw-Hill. 666 p.

22. Zihler A, Gagnon M, Chassard C, Lacroix C. 2011. Protective effects of probiotics on Salmonella infectivity assessed with combined in vitro gut fermentation-cellular models. BMC Microbiol 11:264-277. doi: 10.1186/14712180-11-264

23. Zoccarato I, Barbera S, Tartari E. 1995. Effetto dell'impriego di mangime contente un'associazione antibiótico-probiotico sulle performance del coniglio all'ingrasso. Zootec Nutr Anim 21: 297-304. 